\title{
DQ-Frame Admittance Estimation of Three-Phase Converters with a Wide Range of Operating Points
}

\author{
Hong Gong, Student Member, IEEE, Xiongfei Wang, Senior Member, IEEE \\ and Dongsheng Yang, Senior Member, IEEE
}

\begin{abstract}
The dq-frame admittance of closed-loop controlled three-phase converters is a linearized model that is dependent on the operating points of the system. Yet, it is impractical to measure the converter admittance at all operating points. This paper, thus, proposes an approach to estimating the $d q$-frame admittance of three-phase converters at a wide range of operating points. The method applies multidimensional interpolation to a given set of admittance data, which is measured from the pre-defined operating points. The accuracy of interpolation is then evaluated by using the posterior error estimation method. The number of pre-defined operating points is next adjusted to find a good compromise between the accuracy and efficiency of the approach. Simulations and experimental results verify the effectiveness of the approach.
\end{abstract}

Index Terms-DQ-frame admittance, three-phase converters, admittance estimation, operating points.

\section{INTRODUCTION}

A $\mathrm{s}$ the proportion of power electronics devices in power systems keeps growing in recent years, the stability problems caused by converter-grid interactions have been increasingly reported [1]. Further, grid operators have limited information on the control systems of converters, which makes it difficult to obtain analytical models of converters in practice. Hence, the 'black-box' admittance measurement, which enables to characterize of converters' dynamics without prior knowledge of their control details, is generally preferred in practice for assessing the small-signal stability of the system [2].

Three-phase converters are nonlinear and time-varying dynamical systems, where the nonlinearity is due to the outer power control and synchronization control, and the time variance results from the time-periodic operating trajectories of the ac system [3]. Hence, three-phase converters are generally modelled in the synchronous reference $(d q-)$ frame [4], where the time-varying ac operating trajectories can be transformed as time-invariant (equilibrium) operating points by the Park transformation, and then the linear time-invariant (LTI) model can be obtained by linearizing the converter around the equilibrium operating points in the $d q$-frame [5].

The $d q$-frame admittance measurement is, in essence, a linearization process of three-phase power converters around the equilibrium operating point. The measured admittance model is thus only valid at the specific operating conditions. However, in some cases, e.g. the converters used with renewable energy systems, the operating points vary with the environmental conditions such as wind speed and solar irradiance level [6]. Consequently, the admittance model of converter changes and the measured admittance at a specific operating point may be inadequate for the dynamic analysis. On the other hand, it is impractical to measure the converter admittance at all operating points. It is, therefore, of interest to develop a method for estimating the $d q$-frame admittance of converters over a wide range of operating points.

However, the admittance estimation at different operating points is challenging due to the nonlinear relationship between the converter admittance model and operating points. To address this challenge, a multi-operating-point small-signal modelling method for three-phase converters is reported in [7][10]. In the method, a set of $d q$-frame admittance models at a series of operating points are identified based on the timedomain measurement data. Those models are then multiplied with the nonlinear weight functions, and thus the unmeasured admittance model can be estimated through different weighted sums of the identified admittance models with respect to different operating points. This approach requires using the parametric identification method to obtain the transfer function of admittance models from the time-domain data, which is much more complicated than the frequency-domain measurements due to the pre-selections of the identified model structure and model order [11], [12].

To avoid using the time-domain parametric identification, a multi-operating-point admittance model that is based on the frequency-domain measurement results is recently reported in [13], where the artificial neural network (ANN) is employed to map the nonlinear relationship between the converter admittance model and operating points. However, there is a lack of clear guidance for the ANN training process, i.e., the initialization of ANN structure, the selection for the number of hidden layers, and the number of neurons in each layer, which significantly influences the estimation accuracy and is challenging for the practical application. Further, the multioperating-point admittance model identified from either the time-domain data [7]-[10] or the frequency-domain measurement results [13] requires the utilization of nonlinear weight functions to estimate the unmeasured admittance models through the different weighted combinations of measured admittance models, which makes the approach tedious and complicated.

To avoid using the nonlinear weight functions, an admittance model that is approximated as linearly dependent on operating points is developed [14]. With prior knowledge of the control structure of the converter, the admittance profile is reformulated as a function of operating points and the controller parameters. Given a set of measurement results at different operating points, a group of homogeneous linear equations is obtained to solve unknown control parameters, such that the admittance model can be calculated as a function of constant control parameters and varying operating variables. Yet, the 
method is essentially a linear approximation to the nonlinear relationship between the admittance model and operating points, and its accuracy is highly dependent on the number of measured operating points, yet how to choose the number of measured operating points still remains an open issue.

This paper proposes an admittance estimation method for three-phase converters with a wide range of operating points, where the frequency-domain measurement results of $d q$-frame admittance are first used to formulate a lookup table for a given set of operating points, and the multidimensional interpolation method is then used to estimate the admittance model at the unmeasured operating points. The posterior error estimation method is further used to assess the accuracy of the estimated admittance. The criterion for selecting the number of measured operating points is developed to achieve a good compromise between measurement accuracy and efficiency. Simulations and experimental results validate the accuracy of the approach.

\section{DQ-Frame Admittance Model of Converters}

\section{A.System description}

Fig. 1 illustrates a simplified one-line diagram of a threephase grid-connected Voltage Source Converter (VSC), where $L$ is the filter inductor, and $Z_{\mathrm{g}}$ denotes the grid impedance, including grid capacitor $C_{g}$ and inductor $L_{g} . I_{a b c}$ and $U_{a b c}$ are three-phase inductor currents and three-phase voltages at the Point of Connection ( $\mathrm{PoC})$, respectively. The DC-link voltage control and the AC-bus voltage control are adopted in the outer loops. The inner current control loop and the phase-locked loop (PLL) are also considered. $\theta$ is the phase angle measured by the PLL. $\mathbf{Y}_{d q}$ is the $d q$-frame admittance matrix of VSC, which characterizes the dynamic behaviour at the $\mathrm{PoC}$ of the VSC. Table I provides the main circuit and controller parameters used in this work.

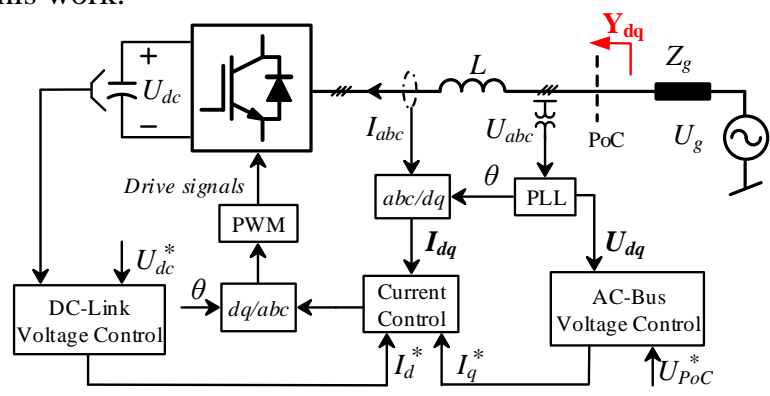

Fig. 1. Simplified one-line diagram of a three-phase grid-connected VSC.

TABLE I

PARAMETERS OF THREE-PHASE VSC

\begin{tabular}{lll}
\hline Symbol & Description & Value \\
\hline$\omega$ & Grid frequency & $314 \mathrm{rad} / \mathrm{s}$ \\
$f_{s}$ & Sampling frequency & $10 \mathrm{kHz}$ \\
$f_{s w}$ & Switching frequency & $10 \mathrm{kHz}$ \\
$U_{d c o}$ & DC-link voltage & $730 \mathrm{~V}$ \\
$T_{d}$ & Dead-time & $2 \mu \mathrm{s}$ \\
$C_{d c}$ & DC capacitor & $1000 \mu \mathrm{F}$ \\
$L$ & Filtered inductor & $3 \mathrm{mH}$ \\
$C_{g}$ & Grid capacitor & $20 \mu \mathrm{F}$ \\
$L_{g}$ & Grid inductor & $7 \mathrm{mH}$ \\
$K_{a c \_p}, K_{a c \_} i$ & AC-bus voltage controller & $0.1 / 10$ \\
$K_{d c \_p}, / K_{d c_{-} i}$ & DC-link voltage controller & $0.1 / 10$ \\
$K_{i \_p}, K_{i} i$ & Current inner controller & $7.85 / 2741.5$ \\
$K_{p}, K_{i}$ & PI controller of PLL & $0.86 / 80.75$ \\
\hline
\end{tabular}

To measure the $d q$-frame admittance of the VSC, the relationships between voltage and current responses at the $d$ and $q$-axes are used, which are given by

$$
\left[\begin{array}{l}
\Delta I_{d} \\
\Delta I_{q}
\end{array}\right]=\mathbf{Y}_{d q}\left[\begin{array}{l}
\Delta U_{d} \\
\Delta U_{q}
\end{array}\right]=\left[\begin{array}{ll}
Y_{d d} & Y_{d q} \\
Y_{q d} & Y_{q q}
\end{array}\right]\left[\begin{array}{c}
\Delta U_{d} \\
\Delta U_{q}
\end{array}\right]
$$

where $\Delta$ denotes the small variations of voltage and current from the equilibrium points. The detailed procedure of the $d q$ frame admittance measurement can be found in [15].

\section{B.Operating point dependence of dq-frame admittance}

For the $d q$-frame admittance model of converters, the operating points are $d q$-axis voltages and currents. Table II lists the variation range of operating points for VSC pre-defined in this work, where the $d$-axis voltage is selected based on the operating conditions of the converter and $d q$-axis currents are determined by the power rating of the converter. Further, the pre-defined operating point range can also be determined by the user's interest. Only the $d$-axis voltage and current are considered for the explanation, yet it can be easily extended to the variations of other variables, e.g. the $q$-axis current.

Fig. 2 illustrates the variation of the $d q$-frame admittance plane with different sets of operating points, where the admittance at a single frequency $(5 \mathrm{~Hz})$ is plotted, and the $q$ axis current is fixed at $5 \mathrm{~A}$. It is clear that both the magnitude and phase values of the admittance terms $Y_{d d}$ and $Y_{q q}$ are highly

TABLE II

OPERATING POINTS OF VSC

\begin{tabular}{|l|l|}
\hline Variables & Variation range \\
\hline$d$-axis voltage $\left(U_{d}\right)$ & $100 \mathrm{~V} \sim 400 \mathrm{~V}$ \\
\hline$d$-axis current $\left(I_{d}\right)$ & $0 \mathrm{~A} \sim 20 \mathrm{~A}$ \\
\hline$q$-axis current $\left(I_{q}\right)$ & $5 \mathrm{~A}$ \\
\hline
\end{tabular}
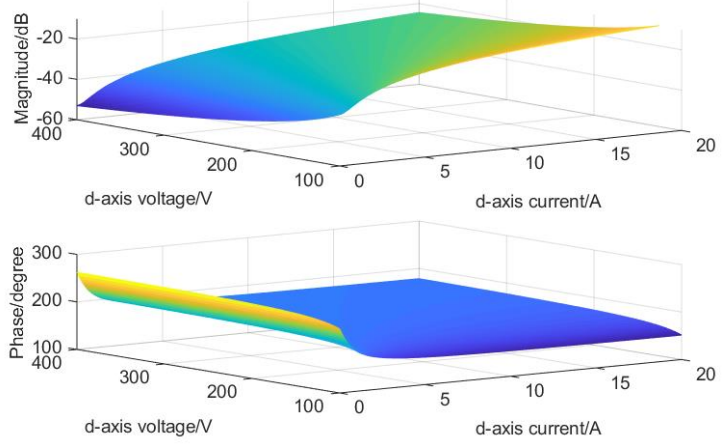

(a)
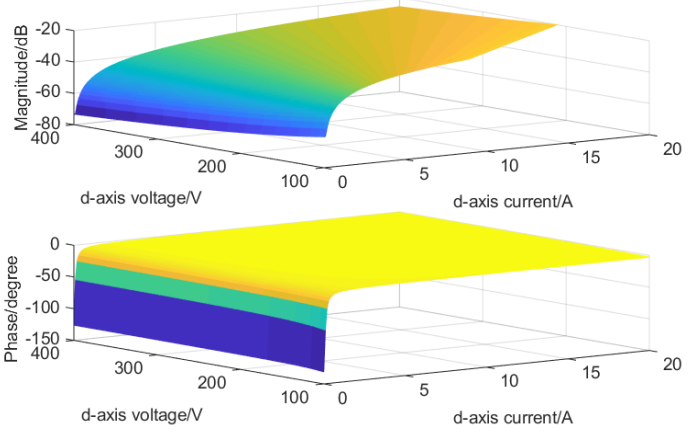

(b)

Fig. 2. Variation of the $d q$-frame admittance at $5 \mathrm{~Hz}$ with different sets of operating points. (a) $Y_{d d}$. (b) $Y_{q q}$. 
dependent on the $d$-axis voltage and current due to the influence of the DC-link voltage controller and PLL. Therefore, the admittance of VSC measured at a specific operating point is inadequate for identifying the worst case of the system.

\section{Proposed Admittance Estimation Method}

Fig. 3 illustrates the procedure of the proposed method for estimating the admittance of VSC at a wide range of operating points, which mainly comprises the lookup-tables formulation, the admittance estimation, and the operating-point interval adjustment. For the defined operating points range, the lookuptables are formulated by storing the measured admittance results for given sets of operating points. The admittance models at the unmeasured operating points are then estimated based on measured admittance results, and the operating-point intervals for the measurement are iteratively adjusted based on the estimation error obtained through the posterior error estimation method to meet the error-index $(\varepsilon)$ requirements.

\section{A. Lookup-tables formulation}

To formulate lookup-tables, the initial operating points for the admittance measurement are set as the maximum and minimum values of the pre-defined operating-point range. The initial interval of operating points is thus defined, and the admittance measurement procedure [15] is then performed to obtain the $d q$-frame admittances at the selected operating points. The measured admittance results are finally stored in the lookup-tables. The lookup-tables will be updated correspondingly when the intervals of measured operating points are re-selected.

The wideband measurement method is adopted in this work, and the admittance matrix of the converter is thus obtained in a wide frequency range within one measurement experiment cycle. Since the admittance matrix of the converter has four elements, i.e. $Y_{d d}, Y_{d q}, Y_{q d}$ and $Y_{q q}$, four lookup-tables are needed to store the measurement data. Further, the operating point consists of multiple variables, as shown in Table II, which makes the lookup-tables multidimensional. Here only the lookup-table for $Y_{d d}$ is discussed for simplicity, yet the lookuptables for other admittance terms can be readily formulated in the same way.

\section{B. Principle of interpolation for admittance estimation}

Fig. 4 illustrates the mathematical description of the admittance estimation for the converter under different operating points at one specific frequency point. Since the relationship between operating points and the converter admittance model is nonlinear, the admittance estimation at different operating points is to find a piecewise linear function $Y_{e}$ to approximate the real admittance $Y$. Thus, the admittance model of the converter at unmeasured operating points can be estimated by using the interpolation method based on the measured admittance results stored in lookup-tables.

In Fig. 2, since two variables are considered as the example, the two-dimensional (bilinear) interpolation method is thus used for the admittance estimation. Fig. 5 (a) illustrates the geometric representation of bilinear interpolation, which is an extension of linear interpolation for interpolating functions of two variables, i.e., $d$-axis voltage $V_{d}$ and current $I_{d}$ on a

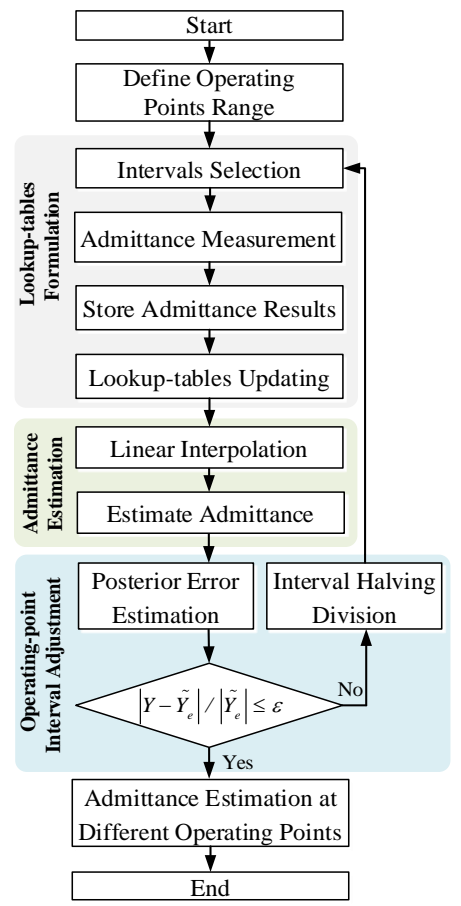

Fig. 3. The procedure of the admittance estimation method at different operating points.

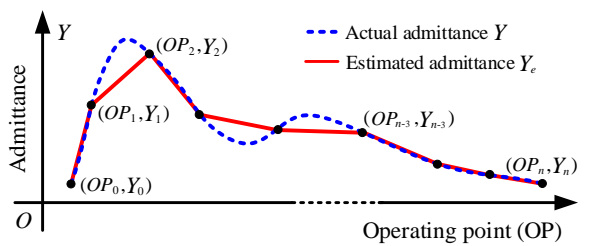

Fig. 4. Mathematical description for admittance estimation under different operating points at one specific frequency point.

rectilinear $2 D$ grid. Given the defined admittance (as shown in Fig. 2), four red dots are the measured admittance results: $Y_{11}=\left(V_{d 1}, I_{d 1}\right), Y_{12}=\left(V_{d 1}, I_{d 2}\right), Y_{21}=\left(V_{d 2}, I_{d 1}\right)$, and $Y_{22}=\left(V_{d 2}, I_{d 2}\right)$, which formulates a lookup-table. To estimate the admittance at the unmeasured operating point $\left(V_{d x}, I_{d x}\right)$, the bilinear interpolation is performed using linear interpolation first in one direction, and then again in the other direction. The result of bilinear interpolation is independent of which axis is interpolated first and which second. The interpolated direction is also not influenced by the admittance results, i.e., the magnitudes and phases.

First, the linear interpolation is performed in the $V_{d^{-}}$ direction. This yields: (blue dots, as shown in Fig. 5)

$$
\begin{gathered}
Y\left(V_{d x}, I_{d 1}\right)=\frac{V_{d 2}-V_{d x}}{V_{d 2}-V_{d 1}} Y_{11}+\frac{V_{d x}-V_{d 1}}{V_{d 2}-V_{d 1}} Y_{21} \\
Y\left(V_{d x}, I_{d 2}\right)=\frac{V_{d 2}-V_{d x}}{V_{d 2}-V_{d 1}} Y_{12}+\frac{V_{d x}-V_{d 1}}{V_{d 2}-V_{d 1}} Y_{22}
\end{gathered}
$$

Second, based on interpolated points (as shown in (2) and (3)), the linear interpolation is further processed in the $I_{d^{-}}$ direction to obtain the estimated admittance: (black dot, as shown in Fig. 5) 


$$
Y\left(V_{d x}, I_{d x}\right)=\frac{I_{d 2}-I_{d x}}{I_{d 2}-I_{d 1}} Y\left(V_{d x}, I_{d 1}\right)+\frac{I_{d x}-I_{d 1}}{I_{d 2}-I_{d 1}} Y\left(V_{d x}, I_{d 2}\right)
$$

The admittance at the unmeasured operating points can be thus estimated by using the linear interpolation method in two directions, respectively. It is more practical to consider three variables, e.g. $d$-axis voltage and $d q$-axis currents. The threedimensional (trilinear) interpolation method is therefore used for the estimation of the admittance models. Fig. $5(b)$ shows the geometric representation of trilinear interpolation, which is also an extension of linear interpolation for interpolating functions of three variables. It is usually performed by using the linear interpolation first in one direction (obtaining blue dots), and then again in another direction (obtaining green dots), and in the last direction (obtaining black dots). Since the principle of the trilinear interpolation is the same as the bilinear interpolation, the detailed derivation will be neglected for simplicity. Moreover, the estimation of the admittance model by using the multidimensional interpolation method can be easily extended when more variables are considered.

Fig. 6 illustrates the variation of the estimated admittance for $Y_{d d}$ at a single frequency point $(5 \mathrm{~Hz})$ with 15 measured operating points. The lookup-table is formulated with measured admittance results. Based on the bilinear interpolation method, the converter admittance model can be estimated by using a group of 4 measured data points stored in the lookup-table. The estimated admittance plane consists of 8 admittance sub-planes, as can be seen from the green plane in Fig. 6.

\section{Operating-Points Intervals AdJustment FOR ADMITTANCE MEASUREMENT}

Fig. 7 shows the comparison between the real admittance model and the estimated admittance model by using different operating point intervals. The number of measured data points determines the accuracy of the estimated admittance model. Obviously, the more data points we used, the more accurate the estimated admittance model will be. Nevertheless, it might need more measurement time and reduce the efficiency of the admittance measurement. There is thus a trade-off between measurement accuracy and efficiency. To manage the accuracy and efficiency holistically, the appropriate intervals for the measured operating points should be selected accordingly. Yet, it is difficult to select a suitable operating-point interval because the admittance model of the converter is unknown before measurement. If the relationship between the estimation error and the number of measured data points can be established, the measurement will then become a closed-loop process and operating-point intervals can be iteratively adjusted according to different accuracy requirements. Further, the specific selection for the accuracy requirements is still an open question. In this work, an error-index $\varepsilon$ is used, which is based on the relative error of the measured admittances.

\section{A. Error estimation of estimated admittance model}

To evaluate the accuracy of the estimated admittance, the error needs to be estimated. According to the one-dimension error estimation [16], the estimation error of two-dimension

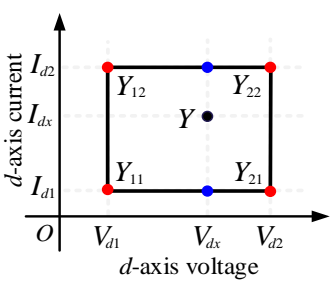

(a)

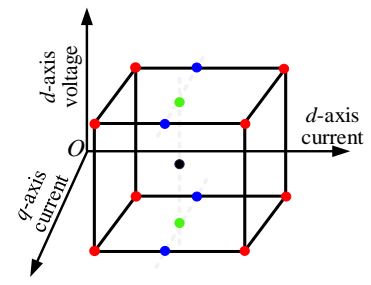

Fig. 5. Geometric representation. (a) Bilinear interpolation. (b) Trilinear interpolation.
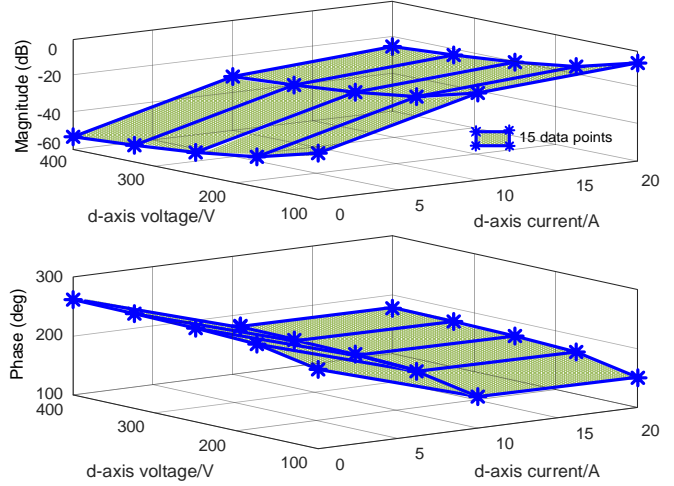

Fig. 6. Variation of the estimated admittance for $Y_{d d}$ at $5 \mathrm{~Hz}$ with different sets of operating points.
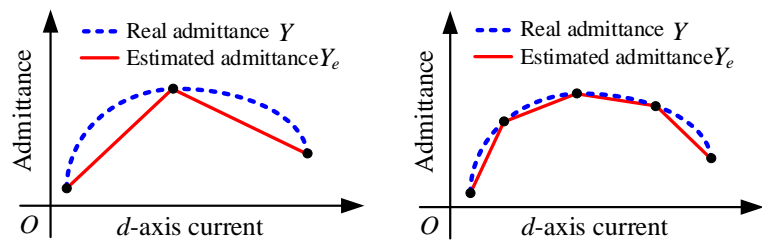

Fig. 7. Comparison between the real admittance and estimated admittance by using different operating point intervals.

interpolation can be approximately obtained based on (4), which can be written as:

$$
\begin{aligned}
R_{e}=Y-Y_{e} \approx & \frac{\partial Y^{\prime \prime}\left(V_{d x}, \tau_{2}\right)}{\partial I_{d x}^{2}}\left(I_{d x}-I_{d 1}\right)\left(I_{d x}-I_{d 2}\right) \\
& +\frac{\partial Y^{\prime \prime}\left(\tau_{1}, I_{d x}\right)}{\partial V_{d x}^{2}}\left(V_{d x}-V_{d 1}\right)\left(V_{d x}-V_{d 2}\right)
\end{aligned}
$$

where $R_{e}$ means the estimation error between the real admittance $Y$ and estimated admittance $Y_{e}$ at the unmeasured operating point $\left(V_{d x}, I_{d x}\right) . \partial Y^{\prime \prime}$ is the second-order partial derivative of $Y . \tau_{1}$ and $\tau_{2}$ represent the arbitrary values within measured operating point ranges. It can be defined as:

$$
V_{d 1} \leq \tau_{1} \leq V_{d 2}, \quad I_{d 1} \leq \tau_{2} \leq I_{d 2}
$$

In the practical application, since the real admittance $Y$ is unknown, the error estimation is impractical by using (5). The posterior error estimation method is therefore used to estimate the error between the real admittance and estimated admittance based on the deviation of the two estimated admittance results.

For two-dimensional interpolation, the error is first estimated in one direction and then again in the other direction. Starting from the $V_{d}$-direction, there is another measured data point $\left(V_{d 3}, Y_{3}\right)$ in the range of the interval $\left[V_{d 1}, V_{d 2}\right]$. While in the $I_{d}$-direction, the $d$-axis current is chosen at the measured data points: $I_{d}=I_{d i}(i=1,2 \ldots)$. Thus, the admittance at the 
unmeasured operating point $\left(V_{d x}, I_{d x}\right)$ can be estimated by using measured data points $\left(V_{d 3}, Y_{3}\right)$ and $\left(V_{d 1}, Y_{1}\right)$. Based on (5), the estimation error can be also calculated as

$$
\begin{aligned}
\tilde{R}_{e} & =Y-\tilde{Y}_{e}-\frac{\partial Y^{\prime \prime}\left(V_{d x}, \tau_{2}\right)}{\partial I_{d x}^{2}}\left(I_{d i}-I_{d 1}\right)\left(I_{d i}-I_{d 2}\right) \\
& \approx \frac{\partial Y^{\prime \prime}\left(\tau_{3}, I_{d i}\right)}{\partial V_{d x}^{2}}\left(V_{d x}-V_{d 1}\right)\left(V_{d x}-V_{d 3}\right), V_{d 1} \leq \tau_{3} \leq V_{d 3}
\end{aligned}
$$

If $\partial Y^{\prime \prime}$ is almost constant [17] in the interval $\left[V_{d 1}, V_{d 3}\right]$, then

$$
\frac{\partial Y^{\prime \prime}\left(\tau_{1}, I_{d i}\right)}{\partial V_{d x}^{2}} \approx \frac{\partial Y^{\prime \prime}\left(\tau_{3}, I_{d i}\right)}{\partial V_{d x}^{2}}
$$

Based on (5), (7) and (8), the estimation +error can be derived in the $V_{d}$-direction as

$$
\tilde{R}_{e}=Y-\tilde{Y}_{e} \approx \frac{V_{d x}-V_{d 3}}{V_{d x}-V_{d 2}}\left(\tilde{Y}_{e}-Y_{e}\right)
$$

where the estimation error $\tilde{R}_{e}$ at the unmeasured operating point $\left(V_{d x}, I_{d x}\right)$ can thus be obtained based on the deviation of the two estimated admittance results $\tilde{Y}_{e}$ and $Y_{e}$.

The estimation error is further calculated in the $I_{d}$-direction. Since the process is the same as the error estimation in the $V_{d^{-}}$ direction, the detailed derivation will be neglected for simplicity.

\section{B. Adjustment criteria of operating-point intervals}

To select appropriate intervals of operating points in twodimensional, the estimation error is first guaranteed to be the minimum in the $V_{d}$-direction and then more data points are selected to make the error in the $I_{d}$-direction to be the minimum.

The interval of the $d$-axis voltage is first adjusted. Assuming that $V_{d i}(i=0,1, \ldots, n)$ is a group of $n_{1}+1$ points which satisfied $a \leq$ $V_{d 0}<V_{d 1}<V_{d 2}<\ldots V_{d n} \leq b$ and distributed on the interval $[a, b]$ equidistantly, then,

$$
h_{1}=(b-a) / n_{1}, \quad V_{d i}=V_{d 0}+i h_{1}
$$

where $h_{1}$ is the operating-point interval of $d$-axis voltage. $a$ and $b$ are the minimum and maximum values within the defined operating-point range, respectively.

If the operating-point interval $h_{1}$ is halved, there are $2 n_{1}+1$ equidistant data points distributed on the interval $[a, b]$. Based on (9) and (10), the maximum estimation error at each subinterval can be derived when $V_{d x}$ is located at the midpoint of subinterval $\left[V_{d i},\left(V_{d i}+V_{d i+1}\right) / 2\right]$. Thus,

$$
\begin{aligned}
\tilde{R}_{e m} & =Y-\tilde{Y}_{e} \approx \frac{1}{3}\left(\tilde{Y}_{e}-Y_{e}\right) \\
& \approx \frac{h_{1}^{2}}{32} \frac{\partial Y^{\prime \prime}\left(\varsigma_{1}, I_{d i}\right)}{\partial V_{d x}^{2}}, V_{d i}<\varsigma_{1}<\frac{V_{d i}+V_{d i+1}}{2}
\end{aligned}
$$

Based on (11), it is noted that the maximum estimation error is becoming smaller with the decreasing of interval $h_{1}$. This proves that the more data points are used, the more accurate the estimated admittance will be.

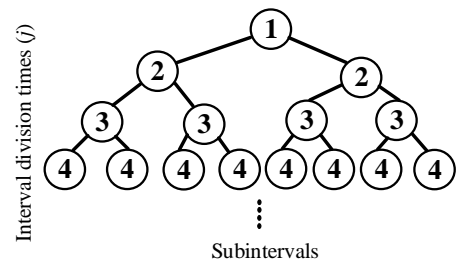

(a)

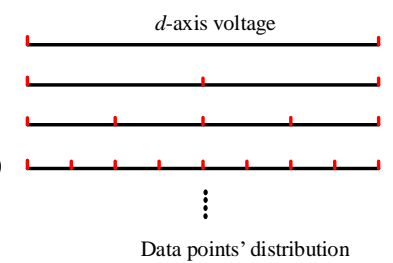

(b)
Fig. 8. Interval selection of operating point with the binary tree structure. (a) Interval division times and the number of subintervals. (b) Data points distribution.

If the deviation of the two-estimated admittance results at all subinterval meets:

$$
\frac{1}{3}\left|\tilde{Y}_{e}-Y_{e}\right| \leq \varepsilon\left|\tilde{Y}_{e}\right|
$$

then $\left|Y-\tilde{Y}_{e}\right| /\left|\tilde{Y}_{e}\right| \leq \varepsilon$, that is to say, the admittance of $\tilde{Y}_{e}$ estimated by using $2 n_{1}+1$ data points in the $V_{d}$-direction meets the accuracy requirements.

On the other hand, if the estimation error is not satisfied with the error-index requirement, the operating-point interval is halved, and more data points need to be measured. With (11) and (12), an iterative process of the interval selection of operating point can be constructed, as shown in Fig. 3, where the intervals will be adjusted constantly until the accuracy requirements are met. In Fig. $8(a)$, the numbers represent interval division times $j$. Fig. $8(b)$ illustrates the data points' distribution and length of subinterval for $d$-axis voltage correspondingly. It is noted that whenever the operating-point interval is halved, the data points and subintervals will be doubled, which is similar to the binary tree in computer programming, so we call it binary tree structure for interval selection of operating points [18].

For the interval selection of $d$-axis current, the same procedure is repeated based on the previous interval selection of $d$-axis voltage. Therefore, the intervals selection for both $d$ axis voltage and current are completed. The intervals selection of operating points can also be easily extended to multidimensional based on the process of two-dimensional interval selection. For simplicity, the detailed process is neglected.

\section{SimULATION AND EXPERIMENT VALIDATION}

\section{A. Simulation Validation}

To validate the accuracy of the admittance estimation method and the effectiveness of the proposed interval selection criteria, an admittance measurement setup is simulated. The PRBS is designed from $1 \mathrm{~Hz}$ to $100 \mathrm{~Hz}$ and its magnitude is chosen as $5 \%$ of the steady value of the voltage. The converter under test is simulated as shown in Fig. 1, and parameters are given in Table I.

Table II has listed the variation range of operating points of the VSC defined in this work, where the variation range of $q$ axis current is added as $0 \mathrm{~A}-20 \mathrm{~A}$ in the simulation validation. Table III shows the numbers of interval division times $j$ and the number of measured data points for $Y_{d d}$ when considering different accuracy requirements for the selection of the operating-point intervals. To satisfy the accuracy requirement, different intervals of the operating points are calculated by 
using the proposed selection criteria and the data points for each variable are equal to $(2 j-1)$. Since the operating-point dependence of $Y_{d d}$ is mainly caused by the DC-link voltage controller, the intervals for both the $d$-axis voltage and $d q$-axis currents have an impact on the admittance estimation.

Fig. 9 shows the comparison results of the estimated admittance planes for $Y_{d d}$ at $5 \mathrm{~Hz}$ by using different errorindexes of $1 \%$ (green plane) and $10 \%$ (red plane). For simplicity, only the intervals of $d$-axis voltage and current are shown. It is noted that more data points are required if a smaller error-index is selected.

The admittance at the operating points $U_{d 0}=225 \mathrm{~V}, I_{d 0}=5 \mathrm{~A}$, $I_{q 0}=5 \mathrm{~A}$, is estimated by using different error-indexes. Fig. 10 shows the comparison between the theoretical admittance and estimated admittance of $Y_{d d}$ based on different error-indexes. It is noted that the estimated admittance is more accurate if a smaller error-index is used while the number of measured data points is increased. This is time-consuming and inefficient. By contrast, the larger the error-index, the more inaccurate the estimated admittances will be. Therefore, there is a trade-off between the efficiency of the measurement and the accuracy of the estimated admittance. On the other hand, in the higher frequency range (above $50 \mathrm{~Hz}$ ), operating points have no impact on the admittance shaping. Thus, with different errorindexes, the same estimated admittance results can be obtained.

TABLE III

NUMBER OF INTERVAL DIVISION TIMES AND DATA POINTS

\begin{tabular}{|l|l|l|}
\hline $\begin{array}{l}\text { Error } \\
\text { Index }(\leq)\end{array}$ & $\begin{array}{l}\text { Number of interval } \\
\text { division times } j\end{array}$ & $\begin{array}{l}\text { Number of } \\
\text { data points }\end{array}$ \\
\hline $1 \%$ & $U_{d}: 3 I_{d}: 2 I_{q}: 2$ & $5 \times 3 \times 3=45$ \\
\hline $5 \%$ & $U_{d}: 2 I_{d}: 1 I_{q}: 1$ & $3 \times 2 \times 2=12$ \\
\hline $10 \%$ & $U_{d}: 1 I_{d}: 1 I_{q}: 1$ & $2 \times 2 \times 2=8$ \\
\hline
\end{tabular}
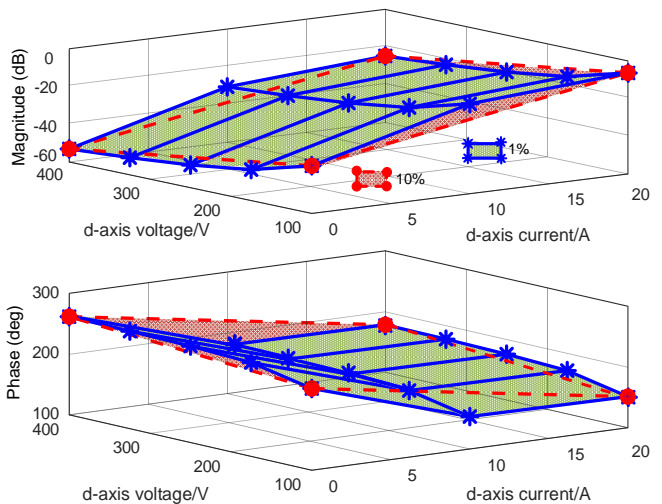

Fig. 9. Comparison of estimated admittances for $Y_{d d}$ at $5 \mathrm{~Hz}$ with different accuracy requirements.
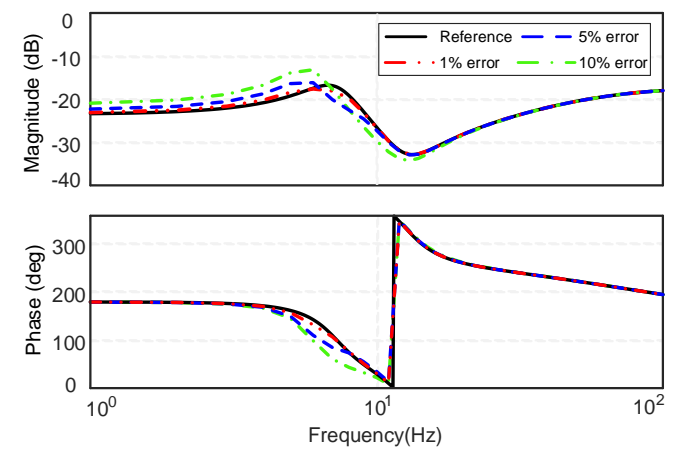

Fig. 10. Comparison between theoretical admittance and estimated admittance of $Y_{d d}$ based on different accuracy requirements.
TABLE IV

NUMBER OF INTERVAL DIVISION TIMES AND DATA POINTS

\begin{tabular}{|l|l|l|}
\hline $\begin{array}{l}\text { Error } \\
\text { Index }(\leq)\end{array}$ & $\begin{array}{l}\text { Number of interval } \\
\text { division times } j\end{array}$ & $\begin{array}{l}\text { Number of } \\
\text { data points }\end{array}$ \\
\hline $1 \%$ & $U_{d}: 3 I_{d}: 1 I_{q}: 2$ & $5 \times 2 \times 3=30$ \\
\hline $5 \%$ & $U_{d}: 2 I_{d}: 1 I_{q}: 1$ & $3 \times 2 \times 2=12$ \\
\hline $10 \%$ & $U_{d}: 1 I_{d}: 1 I_{q}: 1$ & $2 \times 2 \times 2=8$ \\
\hline
\end{tabular}
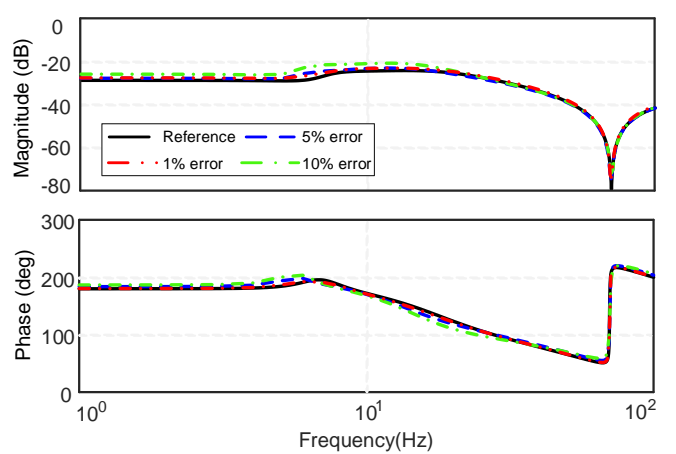

Fig. 11. Comparison between theoretical admittance and estimated admittance of $Y_{d q}$ based on different accuracy requirements.

TABLE V

NUMBER OF INTERVAL Division TIMES AND DATA POINTS

\begin{tabular}{|l|l|l|}
\hline $\begin{array}{l}\text { Error } \\
\text { Index }(\leq)\end{array}$ & $\begin{array}{l}\text { Number of interval } \\
\text { division times } j\end{array}$ & $\begin{array}{l}\text { Number of } \\
\text { data points }\end{array}$ \\
\hline $1 \%$ & $U_{d}: 1 I_{d}: 1 I_{q}: 1$ & $2 \times 2 \times 2=8$ \\
\hline $5 \%$ & $U_{d}: 1 I_{d}: 1 I_{q}: 1$ & $2 \times 2 \times 2=8$ \\
\hline $10 \%$ & $U_{d}: 1 I_{d}: 1 I_{q}: 1$ & $2 \times 2 \times 2=8$ \\
\hline
\end{tabular}

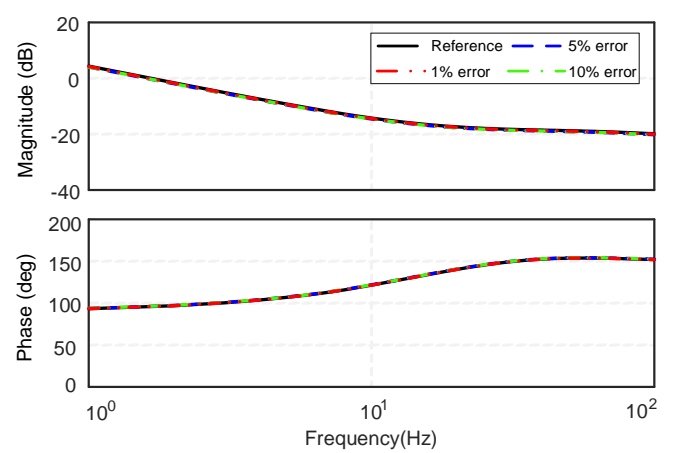

Fig. 12. Comparison between theoretical admittance and estimated admittance of $Y_{q d}$ based on different accuracy requirements.

Table IV shows the numbers of interval division times $j$ and the measured data points for $Y_{d q}$. Fig. 11 illustrates the comparison between the theoretical admittance and estimated admittance of $Y_{d q}$. It can be seen that fewer numbers of measured data points are needed compared with the estimation of $Y_{d d}$. This is because the operating point dependence of this term is mainly introduced by PLL. Therefore, the nonlinear effect is dominated by the $d$-axis voltage and $q$-axis current, which should have more intervals for the accurate admittance estimation.

Table V shows the numbers of interval division times $j$ and the measured data points for $Y_{q d}$. Fig. 12 shows the comparison between the theoretical admittance and estimated admittance of $Y_{q d}$. The same intervals of operating pints are obtained for different accuracy requirements. This is because that the admittance term is linear and not dependent on the operating 
points. Thus, the initial interval of operating points is used to accurately estimate the admittance of $Y_{q d}$.

Table VI shows the numbers of interval division times $j$ and the measured data points for $Y_{q q}$. Fig. 13 illustrates the comparison between the theoretical admittance and estimated admittance of $Y_{q q}$. Due to the nonlinear effect of PLL, a smaller error-index increases the number of interval division times of $d$-axis voltage and current, which can obtain more accurate estimated admittance.

TABLE VI

NUMBER OF INTERVAL Division TIMES AND DATA POINTS

\begin{tabular}{|l|l|l|}
\hline $\begin{array}{l}\text { Error } \\
\text { Index }(\leq)\end{array}$ & $\begin{array}{l}\text { Number of interval } \\
\text { division times } j\end{array}$ & $\begin{array}{l}\text { Number of } \\
\text { data points }\end{array}$ \\
\hline $1 \%$ & $U_{d}: 3 I_{d}: 2 I_{q}: 1$ & $5 \times 3 \times 2=30$ \\
\hline $5 \%$ & $U_{d}: 2 I_{d}: 1 I_{q}: 1$ & $3 \times 2 \times 2=12$ \\
\hline $10 \%$ & $U_{d}: 1 I_{d}: 1 I_{q}: 1$ & $2 \times 2 \times 2=8$ \\
\hline
\end{tabular}
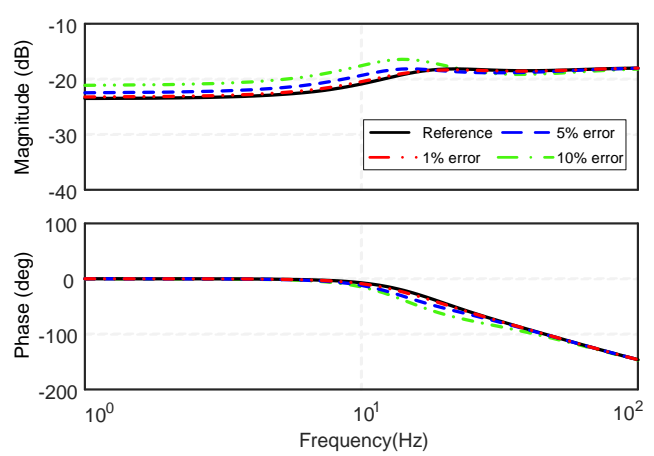

Fig. 13. Comparison between theoretical admittance and estimated admittance of $Y_{q q}$ based on different accuracy requirements.

Due to the influence of different controllers, the estimated intervals of operating points are different for four entries of the admittance matrix to satisfy the different accuracy requirements. The minimum interval for each element is thus selected as the measurement interval to obtain the admittance results which are used for the estimation of admittance at unmeasured operating points.

\section{B. Experiment Validation}

To further verify the correctness of the proposed admittance estimation method at different operating points and the effectiveness of the proposed interval selection criteria, a smallscale prototype based on the proposed admittance measurements setup is built. Yet, the shunt current injection is adopted because this method is much easier to implement for experimental verification.

Fig. 14 shows the detailed experimental setup of the admittance measurement unit. A programmable three-phase voltage source is used to emulate the power grid. Two VSCs are used, where one is considered as the converter under test, the other is used as the source of the perturbation. The current transducer LA 55-P and voltage transducer LV 25-P are used to acquire current and voltage signals for the calculation of the admittance model. The sampling voltage and current are sent to the dSPACE, and the synchronization phase was calculated based on the PLL, and the voltage and current were recorded in the $d q$-domain. In addition, the data would all be processed in the host computer, and the admittance model of the converter would be calculated at the specific operating point.
For the converter under test in the experiment, the parameters are shown in Table I, while the DC-link voltage controller is not adopted for simplicity. The variation range of operating points VSC is also given in Table II.

Table VII shows the numbers of interval division times $j$ and the measured data points for $Y_{d d}$. The admittance at the operating points $U_{d 0}=220 \mathrm{~V}, I_{d 0}=5 \mathrm{~A}, I_{q 0}=5 \mathrm{~A}$, is estimated by using different error-indexes. Fig. 15 shows the comparison between the theoretical admittance and estimated admittance of $Y_{d d}$ based on different error-indexes. Since the DC-link voltage controller is not adopted, the admittance term $Y_{d d}$ is linear and not influenced by different operating points. Therefore, the interval for each variable can be selected as the initial value.

Table VIII shows the numbers of interval division times $j$ and the measured data points for $Y_{d q}$. Fig. 16 illustrates the comparison results of the estimated admittance plane for $Y_{d d}$ at $5 \mathrm{~Hz}$ by using different error-indexes of $5 \%$ (green plane) and $10 \%$ (red plane). For simplicity, only the intervals of $d$-axis voltage and current are shown. Fig. 17 illustrates the comparison between the theoretical admittance and estimated admittance of $Y_{d q}$. It is noted that the interval of $d$-axis voltage significantly influences the accuracy of the estimated admittance due to the nonlinear effects caused by PLL.

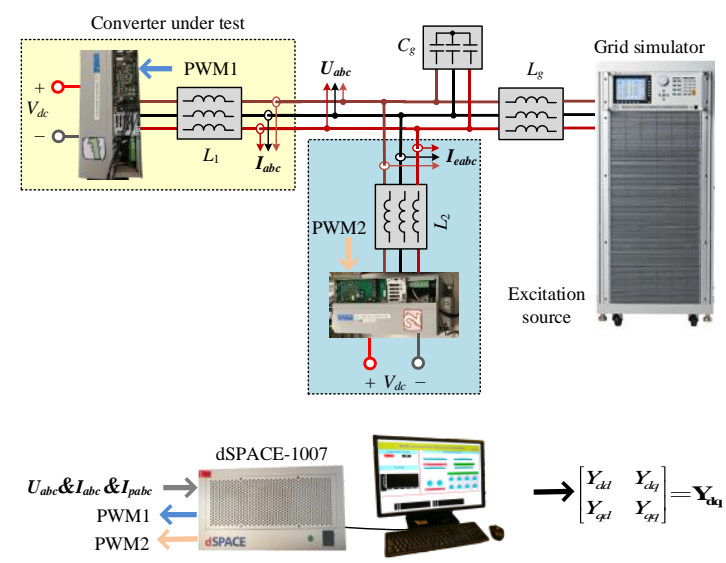

Fig. 14. The small-scale prototype of the admittance measurement.

TABLE VII

NuMBER OF INTERVAL Division TIMES AND DATA POINTS

\begin{tabular}{|l|l|l|}
\hline $\begin{array}{l}\text { Error } \\
\text { Index }(\leq)\end{array}$ & $\begin{array}{l}\text { Number of interval } \\
\text { division times } j\end{array}$ & $\begin{array}{l}\text { Number of } \\
\text { data points }\end{array}$ \\
\hline $5 \%$ & $U_{d}: 1 I_{d}: 1 I_{q}: 1$ & $2 \times 2 \times 2=8$ \\
\hline $10 \%$ & $U_{d}: 1 I_{d}: 1 I_{q}: 1$ & $2 \times 2 \times 2=8$ \\
\hline
\end{tabular}
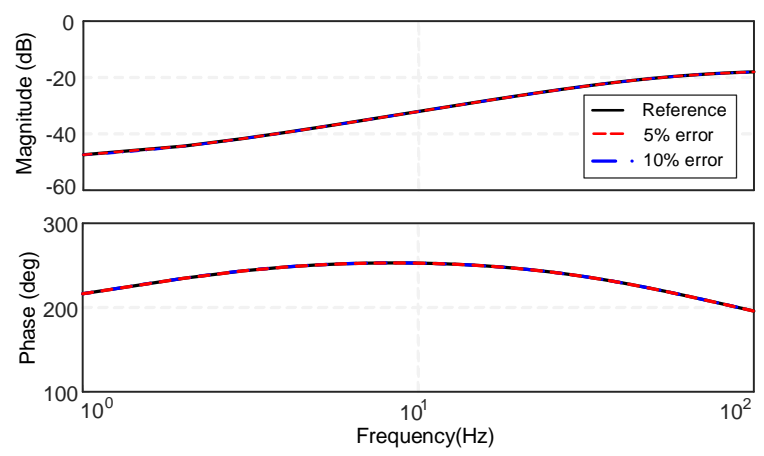

Fig. 15. Comparison between theoretical admittance and estimated admittance of $Y_{d d}$. 
Number of InTERVAl Division Times AND Data Points

\begin{tabular}{|l|l|l|}
\hline $\begin{array}{l}\text { Error } \\
\text { Index }(\leq)\end{array}$ & $\begin{array}{l}\text { Number of interval } \\
\text { division times } j\end{array}$ & $\begin{array}{l}\text { Number of } \\
\text { data points }\end{array}$ \\
\hline $5 \%$ & $U_{d}: 2 I_{d}: 1 I_{q}: 1$ & $3 \times 2 \times 2=12$ \\
\hline $10 \%$ & $U_{d}: 1 I_{d}: 1 I_{q}: 1$ & $2 \times 2 \times 2=8$ \\
\hline
\end{tabular}
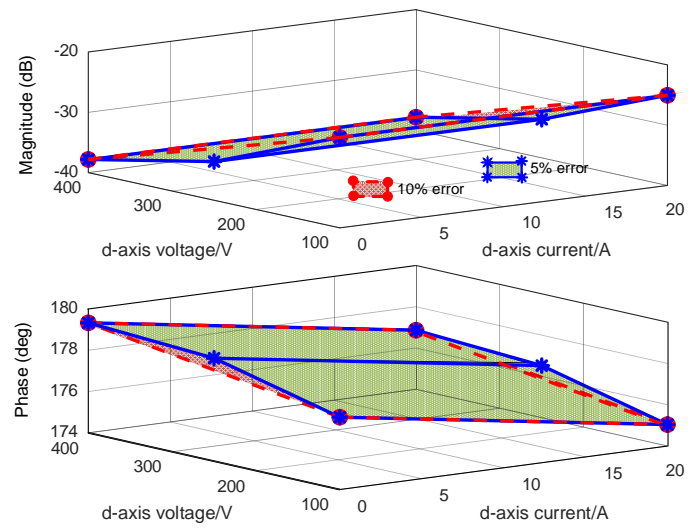

Fig. 16. Comparison of estimated admittances for $Y_{d q}$ at $5 \mathrm{~Hz}$ with different accuracy requirements.
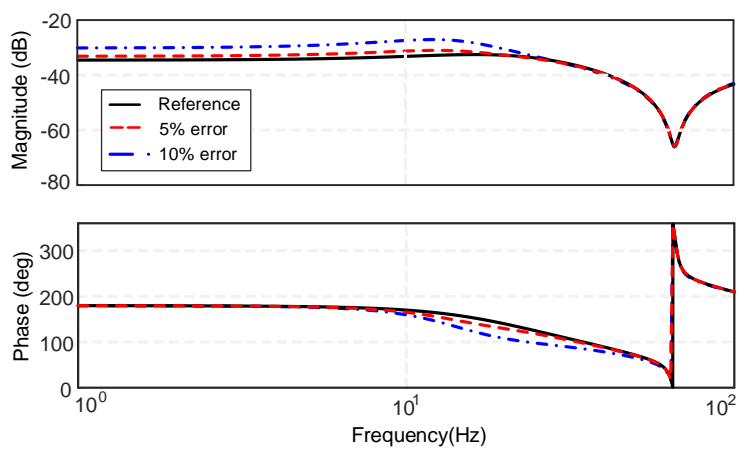

Fig. 17. Comparison between theoretical admittance and estimated admittance of $Y_{d q}$.

TABLE IX

NUMBER OF INTERVAL DIVISION TIMES AND DATA POINTS

\begin{tabular}{|l|l|l|}
\hline $\begin{array}{l}\text { Error } \\
\text { Index }(\leq)\end{array}$ & $\begin{array}{l}\text { Number of interval } \\
\text { division times } j\end{array}$ & $\begin{array}{l}\text { Number of } \\
\text { data points }\end{array}$ \\
\hline $5 \%$ & $U_{d}: 2 I_{d}: 1 I_{q}: 1$ & $3 \times 2 \times 2=12$ \\
\hline $10 \%$ & $U_{d}: 1 I_{d}: 1 I_{q}: 1$ & $2 \times 2 \times 2=8$ \\
\hline
\end{tabular}
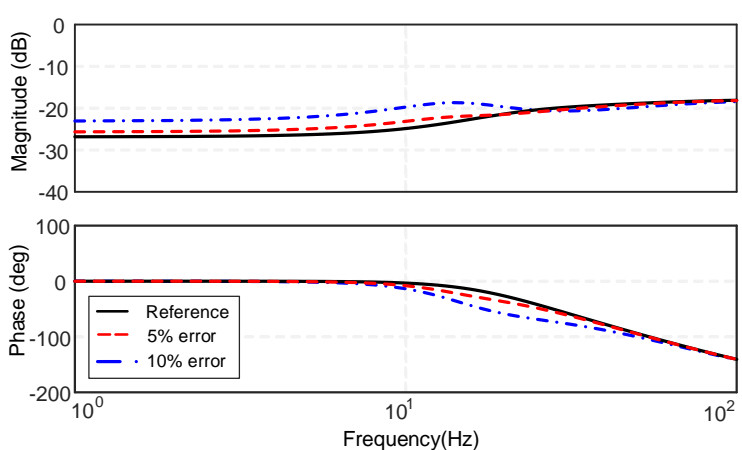

Fig. 18. Comparison between theoretical admittance and estimated admittance of $Y_{q q}$.

Table IX shows the numbers of interval division times $j$ and the measured data points for $Y_{q q}$. Fig. 18 shows the comparison between the theoretical admittance and estimated admittance of $Y_{q q}$. Because of PLL, the $d$-axis voltage needs to be further divided for the accurate estimation of admittance.
The experiment results can be drawn the same conclusion as the simulation results, and they verify the accuracy of the admittance estimation method and the effectiveness of the proposed intervals selection criteria.

\section{CONCLUSION}

This paper has proposed a $d q$-frame admittance estimation method with a wide range of operating points, where the frequency-domain measurement data is first stored in the lookup-tables, and the multidimensional interpolation method is then employed to estimate converter admittances at the unmeasured operating points. To evaluate the accuracy of the estimated admittance models, the posterior error estimation method is adopted, from which a selection criterion has also been proposed to iteratively adjust the operating-point intervals, which provides the guidance for the selection of the number of measured data points and improves the accuracy, reliability, and efficiency of the admittance measurement.

\section{REFERENCES}

[1] F. Blaabjerg, Z. Chen, and S. B. Kjaer, "Power electronics as efficient interface in dispersed power generation systems," IEEE Trans. Power Electron., vol. 19, no. 5, pp. 1184-1194, Sep. 2004.

[2] L. Harnefors, M. Bongiorno and S. Lundberg, "Input-admittance calculation and shaping for controlled voltage-source converters," IEEE Trans. Ind. Electron., vol. 54, no. 6, pp. 3323-3334, Dec. 2007.

[3] X. Wang and F. Blaabjerg, "Harmonic Stability in Power Electronic Based Power Systems: Concept, Modeling, and Analysis," IEEE Trans. Smart Grid. pp.1-1, Mar. 2018.

[4] W. Cao, Y. Ma, L. Yang, F. Wang and L. M. Tolbert, "D-Q admittance based stability analysis and parameter design of three-phase inverterbased AC power systems," IEEE Trans. Ind. Electron., vol. 64, no. 7, pp. 6017-6028, July.2017.

[5] X. Wang, F. Blaabjerg and W. Wu, "Modeling and analysis of harmonic stability in an AC power-electronics-based power system," IEEE Trans. Power Electron., vol. 29, no. 12, pp. 6421-6432, Dec. 2014.

[6] S. Liu, P. X. Liu and X. Wang, "Stochastic small-signal stability analysis of grid-connected photovoltaic systems," IEEE Trans. Ind. Electron., vol.63, no. 2, pp. 1027-1038, Feb. 2016.

[7] V. Valdivia, A. Lazaro, A. Barrado, P. Zumel, C. Fernandez and M. Sanz, "Black-box modeling of three-phase voltage source inverters for systemlevel analysis," IEEE Transactions on Industrial Electronics, vol. 59, no. 9, pp. 3648-3662, Sept. 2012.

[8] V. Valdivia, A. Barrado, A. LÁzaro, P. Zumel, C. Raga and C. FernÁndez, "Simple modeling and identification procedures for "blackbox" behavioral modeling of power converters based on transient response analysis," IEEE Transactions on Power Electronics, vol. 24, no. 12, pp. 2776-2790, Dec. 2009.

[9] V. Valdivia, A. Lázaro, A. Barrado, P. Zumel, C. Fernández and M. Sanz, "Black-box modeling of three phase voltage source inverters based on transient response analysis," in IEEE Applied Power Electronics Conference and Exposition (APEC), Palm Springs, CA, 2010, pp. 12791286

[10] G. Guarderas, A. Francés, R. Asensi and J. Uceda, "Large-signal blackbox behavioral modeling of grid-supporting power converters in AC microgrids," in Proc. IEEE Appl. Power Electron. Conf. (APEC 2017), San Diego, CA, 2017, pp. 153-158.

[11] L. Ljung, System Identification: Theory for the User, $2^{\text {nd }}$ Edition. Englewood Cliffs, Nj: Prentice-Hall, 1999.

[12] I. Rolf; M. Marco. "Identification of Dynamic Systems: An Introduction with Applications. New York, NY, USA: Springer, 2011.

[13] M. Zhang, X. Wang, D. Yang and M. Christensen, "Artificial neural network based identification of multi-operating-point impedance model," IEEE Trans. Power Electron., Early Access, 2020.

[14] W. Liu, X. Xie, J. Shair and X. Li, "A nearly decoupled admittance model for grid-tied VSCs under variable operating conditions," IEEE Trans. Power Electron., vol. 35, no. 9, pp. 9380-9389, Sept. 2020.

[15] J. Huang, K. A. Corzine and M. Belkhayat, "Small-signal admittance measurement of power-electronics-based AC power systems using line- 
to-line current injection", IEEE Trans. Power Electron., vol. 24, no. 2, pp. 445-455, Feb. 2009.

[16] D. Kincaid and W. Cheney, Numerical Analysis, 3rd ed. Providence, RI, USA: American Mathematical Society, 2009, pp. 478-492

[17] H. W. Reddick and F. H. Miller, Advanced Mathematics for Engineers, 3rd Ed. New York, NY, USA: Wiley, 1955.

[18] X. Yue, Z. Fang, F. Wang, Z. Zhang and H. Shi, "A novel adaptive frequency injection method for power electronic system admittance measurement," IEEE Trans. Power Electron., vol. 29, no. 12, pp. 67006711, Dec. 2014. 